University of Texas at El Paso

ScholarWorks@UTEP

$4-2009$

\title{
Expert Knowledge Is Needed for Design under Uncertainty: For p- Boxes, Backcalculation is, in General, NP-Hard
}

Vladik Kreinovich

The University of Texas at El Paso, vladik@utep.edu

Follow this and additional works at: https://scholarworks.utep.edu/cs_techrep

Part of the Computer Engineering Commons

Comments:

Technical Report: UTEP-CS-09-03a

Published in the Proceedings of the 28th North American Fuzzy Information Processing Society Annual Conference NAFIPS'09, Cincinnati, Ohio, June 14-17, 2009.

\section{Recommended Citation}

Kreinovich, Vladik, "Expert Knowledge Is Needed for Design under Uncertainty: For p-Boxes, Backcalculation is, in General, NP-Hard" (2009). Departmental Technical Reports (CS). 28.

https://scholarworks.utep.edu/cs_techrep/28

This Article is brought to you for free and open access by the Computer Science at ScholarWorks@UTEP. It has been accepted for inclusion in Departmental Technical Reports (CS) by an authorized administrator of ScholarWorks@UTEP. For more information, please contact Iweber@utep.edu. 


\title{
Expert Knowledge Is Needed for Design under Uncertainty: For p-Boxes, Backcalculation is, in General, NP-Hard
}

\author{
Vladik Kreinovich \\ Department of Computer Science \\ University of Texas at El Paso \\ 500 W. University \\ El Paso, Texas 89968, USA \\ Email: vladik@utep.edu
}

\begin{abstract}
In engineering design problems, we want to make sure that a certain quantity $c$ of the designed system lies within given bounds - or at least that the probability of this quantity to be outside these bounds does not exceed a given threshold. We may have several such requirements - thus the requirement can be formulated as bounds $\left[\underline{F}_{c}(x), \bar{F}_{c}(x)\right]$ on the cumulative distribution function $F_{c}(x)$ of the quantity $c$; such bounds are known as a $p$-box.

The value of the desired quantity $c$ depends on the design parameters $a$ and the parameters $b$ characterizing the environment: $c=f(a, b)$. To achieve the design goal, we need to find the design parameters $a$ for which the distribution $F_{c}(x)$ for $c=f(a, b)$ is within the given bounds for all possible values of the environmental variables $b$. The problem of computing such $a$ is called backcalculation. For $b$, we also have ranges with different probabilities - i.e., also a p-box. Thus, we have backcalculation problem for p-boxes.

For p-boxes, there exist efficient algorithms for finding a design $a$ that satisfies the given constraints. The next natural question is to find a design that satisfies additional constraints: on the cost, on the efficiency, etc. In this paper, we prove that that without expert knowledge, the problem of finding such a design is computationally difficult (NP-hard). We show that this problem is NP-hard already in the simplest possible linearized case, when the dependence $c=f(a, b)$ is linear. Thus, expert (fuzzy) knowledge is needed to solve design problems under uncertainty.
\end{abstract}

\section{ENGINEERING Design Problems AND the Notion OF BaCkCalculation: Deterministic CASE}

One of the main objective of engineering design is to guarantee that the value of a certain quantity (or several quantities) $c$ is within a given range $[\underline{c}, \bar{c}]$. For example, when we design a car engine, we must make sure:

- that its power is at least as much as needed for the loaded car to climb the steepest mountain roads,

- that the concentration of undesirable substances in the exhaust does not exceed the required threshold, etc.

The value of the quantity $c$ usually depends on the parameters $a$ describing the design and on the parameters $b$ of the environment: $c=f(a, b)$. For example, the concentration of a substance in a car exhaust depends:
- on the parameter(s) that describe the design of the car exhaust filters, and

- on the concentration of the chemicals in the original fuel.

We need to select a design $a$ in such a way that

$$
c=f(a, b) \in[\underline{c}, \bar{c}]
$$

for all possible values of the environmental parameter(s) $b$.

In this paper, we consider the simplest case when:

- the design of each system is characterized by a single parameter $a$, and

- the environment is also characterized by a single parameter $b$.

We will show that already in this simple case, the design problem is computationally difficult (NP-hard).

It is well known that expert knowledge can help in solving NP-hard problems. For example, in general, the problem of controlling a system is, in general, NP-hard. However, for systems for which human controllers have expertise of controlling them, intelligent control techniques can transform this expertise into successful automatic control algorithms [5], [6].

Thus, to efficiently solve design problem under uncertainty, we must use expert knowledge - the knowledge for which fuzzy technique have been invented; see, e.g., [5], [6].

To be able to find a design that satisfies the given constraint on $c$ for all possible values of the environmental parameter $b$, we need to know which values of $b$ are possible, i.e., we need to know the range $[\underline{b}, \bar{b}]$ of possible values of $b$. Thus, we arrive at the following problem:

- we know the desired range $[\underline{c}, \bar{c}]$;

- we know the dependence $c=f(a, b)$;

- we know the range $[\underline{b}, \bar{b}]$ of possible values of $b$;

- we want to describe the set of all values of $a$ for which $f(a, b) \in[\underline{c}, \bar{c}]$ for all $b \in[\underline{b}, \bar{b}]$.

This design-related problem is sometimes called a backcalculation problem, to emphasize its difference from the forward calculation problem, when 
- we are given a design $a$ and

- we want to estimate the value of the desired characteristic $c=f(a, b)$.

\section{LINEARIZED PROBLEM}

In many engineering situations, the intervals of possible values of $a$ and $b$ are reasonably narrow: $a \approx \widetilde{a}$ and $b \approx \widetilde{b}$ for some $\widetilde{a}$ and $\widetilde{b}$. In such situations, we can expand the dependence $c=f(a, b)$ in Taylor series and ignore terms which are quadratic and higher order in terms of $\Delta a \stackrel{\text { def }}{=} a-\widetilde{a}$ and $\Delta b \stackrel{\text { def }}{=} b-\widetilde{b}$. As a result, we get a simple linear dependence

$$
c \approx c_{0}+k_{a} \cdot a+k_{b} \cdot b .
$$

We can simplify this expression even further if we take into account that the numerical value of each of the quantities $a$ and $b$ depends on the choice of the starting point and on the choice of a measuring unit. If we change the starting point and the measuring unit, then the new numerical value can be obtained from the original one by an appropriate linear transformation. For example, if we know the temperature $t_{C}$ in Celsius, then we can compute the temperature $t_{F}$ in the Fahrenheit scale as $t_{F}=32+1.8 \cdot t_{C}$. We can use this possibility to simplify the above expression for $c$. Specifically, we can change the starting points and the measuring units in such a way that:

- the new numerical value for $a$ is described by the linear expression $c_{0}+k_{a} \cdot a$, and

- the new numerical value for $b$ is described by the linear expression $k_{b} \cdot b$.

In these new scales, the dependence of $c$ on $a$ and $b$ takes the simplest form $c=a+b$.

We will show that the design problem becomes computationally difficult (NP-hard) already for this simplest case.

\section{From Guaranteed Bounds to P-BoXes}

Ideally, it is desirable to provide a $100 \%$ guarantee that the quantity $c$ never exceeds the threshold $\bar{c}$. In practice, however, too many unpredictable factors affect the performance of a system and thus, such a guarantee is not realistically possible. What we can realistically guarantee is that the probability of exceeding $c$ is small enough. In other words, we set some threshold $\varepsilon_{c}>0$ and we require that $\operatorname{Prob}(c \leq \bar{c}) \geq 1-\varepsilon_{c}$.

In addition to this requirement, we can also require that the excess of $c$ over $\bar{c}$ be not too large. This can be done, e.g., by requiring that for some value $c_{1}>\bar{c}$, the probability $\operatorname{Prob}\left(c \leq \bar{c}_{1}\right)$ is bounded from below by the value $1-\varepsilon_{1}$ for some smaller $\varepsilon_{1}<\varepsilon$. We can several such requirements for different values $c_{i}$ and $\varepsilon_{i}$.

Similarly, instead of the idealized exact inequality $c \geq \underline{c}$, in practice, we can only require that $\operatorname{Prob}(c \geq \underline{c}) \leq \delta$ for some small probability $\delta>0$.

From the mathematical viewpoint, all such constraints are lower or upper bounds on the values of the cumulative distribution function $F_{c}(x) \stackrel{\text { def }}{=} \operatorname{Prob}(c \leq x)$. By combining the bounds corresponding to all the constraints, we can thus conclude that the cdf $F_{c}(x)$ must satisfy, for every $x$, the inequalities

$$
\underline{F}_{c}(x) \leq F_{c}(x) \leq \bar{F}_{c}(x),
$$

where $\underline{F}_{c}(x)$ is the largest of all the lower bounds on $F_{c}(x)$ and $\bar{F}_{c}(x)$ is the smallest of all the upper bounds on $F_{c}(x)$.

In other words, for every $x$, the corresponding value $F_{c}(x)$ must belong to the interval $\left[\underline{F}_{c}(x), \bar{F}_{c}(x)\right]$. This $x$-dependent interval is known as a probability box, or a $p$-box, for short; see, e.g., [2].

Similarly, for the environmental parameter $b$, we rarely know guaranteed bounds $\underline{b}$ and $\bar{b}$. At best, we know that for a given bound $\bar{b}$, the probability of exceeding this bound is small, i.e., that $\operatorname{Prob}(b \leq \bar{b}) \geq 1-\varepsilon_{b}$ for some small $\varepsilon_{b}$. So here too, instead of a single bound, in effect, we have a p-box $\left[\underline{F}_{b}(x), \bar{F}_{b}(x)\right]$.

\section{TOWARds Formulating THE DESIGN (BACKCALCUlATION) PROBLEM FOR P-BoXES}

In the deterministic approach to design, we assume that we can manufacture an object with the exact value $a$ of the corresponding parameter - or at least the value which is guaranteed to be within the given bounds $[\underline{a}, \bar{a}]$.

In manufacturing, however, it is not practically possible to always guarantee that the value $a$ is within the given interval. At best, we can guarantee that, e.g., the probability of $a \leq \bar{a}$ is greater than or equal to $1-\varepsilon_{a}$ for some small value $\varepsilon_{a}$. In other words, the design restriction on $a$ can also be formulated in terms of p-boxes. Thus, we arrive at the following problem.

\section{BACKCALCUlation PROBLEM FOR P-BOXES}

We are given:

- the desired p-box $\left[\underline{F}_{c}(x), \bar{F}_{c}(x)\right]$ for $c$;

- the dependence $c=f(a, b)$; and

- the p-box $\left[\underline{F}_{b}(x), \bar{F}_{b}(x)\right]$ describing $b$.

Our objective is to find a p-box $\left[\underline{F}_{a}(x), \bar{F}_{a}(x)\right]$ for which:

- for every probability distribution

$$
F_{a}(x) \in\left[\underline{F}_{a}(x), \bar{F}_{a}(x)\right],
$$

- for every probability distribution $F_{b}(x) \in\left[\underline{F}_{b}(x), \bar{F}_{b}(x)\right]$, and

- for all possible correlations between $a$ and $b$,

the distribution of $c=f(a, b)$ is within the given p-box $\left[\underline{F}_{c}(x), \bar{F}_{c}(x)\right]$.

\section{REMINDER: FORWARD CALCULATION FOR P-BOXES}

In order to analyze the backcalculation problem for p-boxes, let us first describe how the corresponding forward calculation problem is solved. Let us assume that we know:

- the p-box $\left[\underline{F}_{a}(x), \bar{F}_{a}(x)\right]$ for $a$; and

- the p-box $\left[\underline{F}_{b}(x), \bar{F}_{b}(x)\right]$ describing $b$.

The objective of forward calculation is to find the range $\left[\underline{F}_{c}(x), \bar{F}_{c}(x)\right]$ of possible values of $F_{c}(x)$ for $c=f(a, b)$ for all possible distributions $F_{a}(x) \in\left[\underline{F}_{a}(x), \bar{F}_{a}(x)\right]$ and $F_{b}(x) \in\left[\underline{F}_{b}(x), \bar{F}_{b}(x)\right]$ and all possible correlations between $a$ and $b$. 
It turns out that these calculations are best done in terms not of the original cdfs and p-boxes, but rather in terms of their inverses - quantile functions. For a cdf $F_{a}(x)$, quantiles $a_{0}, \ldots, a_{n}$ are described as values for which $F_{a}\left(a_{i}\right)=\frac{i}{n}$. Since the cdf is monotonic, the quantiles are also monotonic: if $i<j$, then $a_{i} \leq a_{j}$.

When instead of the exact cdf, we only know a p-box $\left[\underline{F}_{a}(x), \bar{F}_{a}(x)\right]$, then instead of the exact quantiles $a_{i}$ we only know interval bounds $\left[\underline{a}_{i}, \bar{a}_{i}\right]$ for these quantiles:

- the lower bounds $\underline{a}_{i}$ are quantiles of the function $\bar{F}_{c}(x)$, and

- the upper bounds $\bar{a}_{i}$ are quantiles of the function $\underline{F}_{c}(x)$. These bounds are also monotonic: if $i<j$, then $\underline{a}_{i} \leq \underline{a}_{j}$ and $\bar{a}_{i} \leq \bar{a}_{j}$.

For the function $c=f(a, b)=a+b$, forward calculation can be easily described in terms of the quantile bounds: once we know the bounds $\left[\underline{a}_{i}, \bar{a}_{i}\right]$ and $\left[\underline{b}_{i}, \bar{b}_{i}\right]$ corresponding to $a$ and $b$, we can compute the quantile bounds for $c$ as follows:

$$
\begin{gathered}
\underline{c}_{i}=\max _{j}\left(\underline{a}_{j}+\underline{b}_{i-j}\right) ; \\
\bar{c}_{i}=\min _{j}\left(\bar{a}_{j-i}+\bar{b}_{n-j}\right) .
\end{gathered}
$$

These formulas were first described in [9].

\section{Formulation of THE PROBlem}

In terms of quantile bounds, the backcalculation problem takes the following form:

- we know the quantile intervals $\left[\underline{b}_{i}, \bar{b}_{i}\right]$ corresponding to the environmental variable $b$;

- we are given the intervals $\left[\widetilde{c}_{i}, \widetilde{\bar{c}}_{i}\right]$ that should contain the quantiles for $c=a+b$;

- we must find the bounds $\underline{a}_{i}$ and $\bar{a}_{i}$ for which, for the values $\underline{c}_{i}$ and $\bar{c}_{i}$ are determined by the formulas (3) and (4), we have $\left[\underline{c}_{i}, \bar{c}_{i}\right] \subseteq\left[\widetilde{c}_{i}, \widetilde{\bar{c}}_{i}\right]$.

\section{In EFFECT, We HaVE Two DifFERENT Problems: FINDING $\underline{a}_{i}$ AND FINDING $\bar{a}_{i}$}

An important consequence of the formulas (3) and (4) is that:

- the lower bounds $\underline{c}_{i}$ for $c$ are determined only by the lower bounds $\underline{a}_{i}$ and $\underline{b}_{i}$ for $a$ and $b$, and

- the upper bounds $\bar{c}_{i}$ for $c$ are determined only by the upper bounds $\bar{a}_{i}$ and $\bar{b}_{i}$ for $a$ and $b$.

Thus, in effect, we can formulate the problem of finding the values $\underline{a}_{i}$ and the problem of finding the values $\bar{a}_{i}$ as two separate yet similar problems.

Without losing generality, in the following text, we will only consider the following problem of finding $\underline{a}_{i}$ :

- we know the values $\underline{b}_{i}$;

- we are given the values $\widetilde{c}_{i}$;

- we must find the values $\underline{a}_{0} \leq \ldots \leq \underline{a}_{n}$ for which

$$
\widetilde{c}_{i} \leq \max _{j}\left(\underline{a}_{j}+\underline{b}_{i-j}\right)
$$

\section{A Designed System Usually Consists of SEVERAL SUbSYSTEMS}

A designed system usually consists of several subsystems. So, instead of selecting a single p-box for a single design parameter $a$, we need to design p-boxes corresponding to all these subsystems.

Let $S$ denote the number of these subsystems, and let $\underline{a}_{i}^{(s)}$, $\underline{b}_{i}^{(s)}$, and $\widetilde{\bar{c}}_{i}^{(s)}$ denote quantile bounds corresponding to the $s$-th subsystem, $s=1,2, \ldots, S$.

Thus, we arrive at the following problem:

- we know the values $\underline{b}_{i}^{(s)}$;

- we are given the values $\widetilde{c}_{i}^{(s)}$;

- we must find, for each $s=1, \ldots, S$, the values

$$
\underline{a}_{0}^{(s)} \leq \ldots \leq \underline{a}_{n}^{(s)}
$$

for which

$$
\underline{\underline{c}}_{i}^{(s)} \leq \max _{j}\left(\underline{a}_{j}^{(s)}+\underline{b}_{i-j}^{(s)}\right) .
$$

\section{There Exist Effective Algorithms for BACKCALCULATION}

For p-boxes, there are efficient algorithms for solving the backcalculation problem; see, e.g., [1], [3], [4], [8].

\section{NeEd For Additional Cost Constraints}

In general, as we can see, the backcalculation problem has many possible solutions. Some design solutions require less efforts, some require more efforts. It is therefore desirable not just to find a solution, but rather to find a solution which satisfies given constraints on the manufacturing efforts such as cost, energy expenses, etc.

The values $\underline{a}_{i}^{(s)}$ are the lower bounds on the design parameters. The smaller the lower bounds, the easier it is to maintain them. Thus, the cost of maintaining a lower bound increases with the value $\underline{a}_{i}^{(s)}$.

In this paper, we show that the problem is NP-hard even for the simplest case when the corresponding effort is simply proportional to the value $\underline{a}_{i}^{(s)}$, and thus, the overall effort of maintaining all the characteristics $\underline{a}_{i}^{(s)}$ is equal to the weighted linear combination

$$
E=\sum_{s=1}^{S} \sum_{i=0}^{n} w_{i}^{(s)} \cdot \underline{a}_{i}^{(s)} .
$$

The corresponding constraint is that this effort should not exceed a given value $e$.

As we have mentioned, we may have several $(C)$ constraints corresponding to different type of effort - cost, energy consumption, etc. Thus, in general, the constrained backcalculation problem takes the following form. 


\section{XiI. Formulation of the Problem in Precise MATHEMATICAL TERMS}

We are given:

- positive integers $n, S$, and $C$;

- the values $\underline{b}_{i}^{(s)}$ corresponding to different $s=1, \ldots, S$ and $i=0, \ldots, n$;

- the values $\widetilde{c}_{i}^{(s)}$ corresponding to different $s=1, \ldots, S$ and $i=0, \ldots, n$;

- the values $e_{c}$ corresponding to different $c=1, \ldots, C$, and

- the values $w_{c, i}^{(s)}$ corresponding to different $s=1, \ldots, S$, $c=1, \ldots, C$, and $i=0, \ldots, n$.

We must find for each $s=1, \ldots, S$, the values

$$
\underline{a}_{0}^{(s)} \leq \ldots \leq \underline{a}_{n}^{(s)}
$$

for which the following two sets of inequalities are satisfied:

$$
\begin{gathered}
\underline{\tilde{c}}_{i}^{(s)} \leq \max _{j}\left(\underline{a}_{j}^{(s)}+\underline{b}_{i-j}^{(s)}\right) ; \\
\sum_{s=1}^{S} \sum_{i=0}^{n} w_{c, i}^{(s)} \cdot \underline{a}_{i}^{(s)} \leq e_{c} .
\end{gathered}
$$

\section{OUR MAIN RESUlt}

Our main result is that the above problem is NP-hard.

\section{Proof}

Main idea. Formally, NP-hard means that an arbitrary problem from a certain class NP can be reduced to this problem; see, e.g., [7]. Thus, to prove that a problem is NP-hard, it is sufficient to prove that a known NP-hard problem can be reduced to it. Indeed,

- by definition of NP-hardness, every problem with the class NP can be reduced to the known NP-hard problem;

- since this known problem can be reduced to our problem,

- we can therefore conclude that every problem form the class NP can be reduced to our problem;

- in other words, we can conclude that our problem is NPhard.

In our proof, as such a known NP-hard problem, we take the knapsack problem; see, e.g., [7]. In this problem, we are given a set of $S$ objects, for each of which we know its volume $v_{s}>0$ and its price $p_{s}>0$. We also know the total volume $V$ of a knapsack and the threshold price $P$. Within the restriction on the volume, we must select some of the $S$ objects in such a way that the total price of all the selected objects is at least $P$.

To describe this problem in precise terms, for each object $i$, we define a new variable $x_{s}$ such that $x_{s}=1$ if the $s$-th object is taken and $x_{s}=0$ if the $s$-th object is not taken. In terms of these new variables, the overall volume of all the selected objects is equal to $\sum_{s=1}^{S} v_{s} \cdot x_{s}$, and the overall price of all the selected objects is equal to $\sum_{s=1}^{S} p_{s} \cdot x_{s}$. Thus, the knapsack problem takes the following form: find the values $x_{s} \in\{0,1\}$ for which

$$
\sum_{s=1}^{S} v_{s} \cdot x_{s} \leq V
$$

and

$$
\sum_{s=1}^{S} p_{i} \cdot x_{s} \geq P
$$

We will prove that this problem can be reduced to the above backcalculation problem, i.e., that for each instance $v_{1}, \ldots, v_{S}, p_{1}, \ldots, p_{S}, V, P$ of the knapsack problem there is an instance of the backcalculation problem whose solution can effectively lead to the solution of the original knapsack problem.

Towards reduction: selection of p-boxes. In our reduction, we will use the same pair of p-boxes $b, c$ for all $S$ subsystems, the only difference will be in the weights.

Let us denote the common value of $\underline{b}_{i}^{(s)}$ for all $s=1, \ldots, S$ by $b_{i}$ and the common value of $\underline{\widetilde{c}}_{i}^{(s)}$ by $c_{i}$. In these notations, the constrains on the unknowns $\underline{a}_{i}^{(s)}$ take a simplified form

$$
c_{i} \leq \max _{j}\left(\underline{a}_{j}^{(s)}+b_{i-j}\right) .
$$

In our reduction, we take $n=1$. For $n=1$, for every $s$, the inequalities (12) lead to the following constraints on the corresponding two unknown $\underline{a}_{0}^{(s)} \leq \underline{a}_{1}^{(s)}$ :

$$
\begin{gathered}
c_{0} \leq \underline{a}_{0}^{(s)}+b_{0} ; \\
c_{1} \leq \max \left(\underline{a}_{0}^{(s)}+b_{1}, \underline{a}_{1}^{(s)}+b_{0}\right) .
\end{gathered}
$$

Specifically, we take $b_{0}=c_{0}=0, b_{1}=1$, and $c_{1}=2$. For these values, the above inequalities take the following form:

$$
\begin{gathered}
\underline{a}_{0}^{(s)} \geq 0 ; \\
2 \leq \max \left(\underline{a}_{0}^{(s)}+1, \underline{a}_{1}^{(s)}\right) .
\end{gathered}
$$

The largest of the two values is greater than or equal to 2 if and only if (at least) one of these two values is greater than or equal to 2 . Thus, the second constraint (16) means that:

- either $2 \leq \underline{a}_{0}^{(s)}+1$ and thus $\underline{a}_{0}^{(s)} \geq 1$

- or $\underline{a}_{1}^{(s)} \geq 2$.

Analysis of the selected p-boxes. Let us show that out of all possible solutions $\underline{a}_{0}^{(s)} \leq \underline{a}_{1}^{(s)}$ satisfying these two inequalities, only two solutions satisfy the additional constraint $\underline{a}_{0}^{(s)}+\underline{a}_{1}^{(s)} \leq 2$ :

- $\underline{a}_{0}^{(s)}=\underline{a}_{1}^{(s)}=1$ and

- $\underline{a}_{0}^{(s)}=0$ and $\underline{a}_{1}^{(s)}=2$.

Indeed, we know that for each solution, we have:

- either $\underline{a}_{0}^{(s)} \geq 1$

- or $\underline{a}_{1}^{(s)} \geq 2$.

In the first case $\underline{a}_{0}^{(s)} \geq 1$, we have

$$
\underline{a}_{0}^{(s)}+\underline{a}_{1}^{(s)}=2 \underline{a}_{0}^{(s)}+\left(\underline{a}_{1}^{(s)}-\underline{a}_{0}^{(s)}\right) .
$$


The first term in the right-hand side is $\geq 2$, the second term is always non-negative - since $\underline{a}_{1}^{(s)} \geq \underline{a}^{(s)}$. Thus, the only possibility for the right-hand side sum to be $\leq 2$ is when the value $2 \underline{a}_{0}^{(s)}$ is exactly 2 , and the difference $\underline{a}_{1}^{(s)}-\underline{a}_{0}^{(s)}$ is exactly 0 . In this case, we have $\underline{a}_{0}^{(s)}=\underline{a}_{1}^{(s)}=1$.

In the second case $\underline{a}_{1}^{(s)} \geq 2$, due to $\underline{a}_{0}^{(s)} \geq 0$ (condition (15)), the only possibility for the sum $\underline{a}_{0}^{(s)}+\underline{a}_{1}^{(s)}$ to be $\leq 2$ is when $\underline{a}_{0}^{(s)}=0$ and $\underline{a}_{1}^{(s)}=2$.

Reduction and the final part of the proof. We will reduce the given instance of the knapsack problem to the following system with 3 constraints:

- In the first constraint, we take $w_{1, i}^{(s)}=1$ for all $s$ and $i$, and we take $e_{1}=2 \cdot S$.

- In the second constraint, we take $w_{2,0}^{(s)}=v_{s}, w_{2,1}^{(s)}=0$, and $e_{2}=V$.

- In the third constraint, we take $w_{2,0}^{(s)}=0, w_{2,1}^{(s)}=p_{s}$, and

$$
e_{3}=P-2 \cdot \sum_{s=1}^{S} p_{s}
$$

Let us first analyze the first constraint

$$
\sum_{s=1}^{S}\left(\underline{a}_{0}^{(s)}+\underline{a}_{1}^{(s)}\right) \leq 2 \cdot S .
$$

As we have shown in the previous section, each simple sum $\underline{a}_{0}^{(s)}+\underline{a}_{1}^{(s)}$ is at least 2 , and it is only equal to 2 when either $\underline{a}^{(s)}=0$ or $\underline{a}_{0}^{(s)}=1$. Thus, the only possibility for the sum $\sum_{s=1}^{S}\left(\underline{a}_{0}^{(s)}+\underline{a}_{1}^{(s)}\right)$ of $S$ such simple sums to not exceed $2 S$ is when each of these sums is equal to exactly 2 , i.e., when for every $s$,

- either either $a^{(s)}=0$ or $\underline{a}_{0}^{(s)}=1$, and

- $\underline{a}_{1}^{(s)}=2-\underline{a}_{0}^{(s)}$.

For these values $\underline{a}_{i}^{(s)}$, the second constraint takes the form

$$
\sum_{s=1}^{S} v_{s} \cdot \underline{a}_{0}^{(s)} \leq V
$$

and the third constraint takes the form

$$
\sum_{s=1}^{S} p_{s} \cdot \underline{a}_{1}^{(s)} \leq 2 \cdot \sum_{s=1}^{S} p_{s}-P .
$$

Substituting the expression $\underline{a}_{1}^{(s)}=2-\underline{a}_{0}^{(s)}$ into this inequality, we get

$$
\sum_{s=1}^{S} p_{s} \cdot\left(2-\underline{a}_{0}^{(s)}\right) \leq 2 \cdot \sum_{s=1}^{S} p_{s}-P
$$

i.e., equivalently,

$$
2 \sum_{s=1}^{S} p_{s}-\sum_{s=1}^{S} p_{s} \cdot \underline{a}_{0}^{(s)} \leq 2 \sum_{s=1}^{S} p_{s}-P,
$$

which, in its turn, is equivalent to

$$
\sum_{s=1}^{S} p_{s} \cdot \underline{a}_{0}^{(s)} \geq P,
$$

Thus, for every solution to the constrained backcalculation problem, the values $x_{s} \stackrel{\text { def }}{=} \underline{a}_{0}^{(s)}$ form a solution to the knapsack problem: each of them is equal to 0 or 1 , and they satisfy the corresponding inequalities (20) and (24).

Vice versa, one can easily check that if the values $x_{s}$ form a solution to the knapsack problem, then the corresponding values $\underline{a}_{0}^{(s)}=x_{s}$ and $\underline{a}_{1}^{(s)}=2-x_{s}$ form a solution to the constrained backcalculation problem.

The reduction is proven, so the backcalculation problem is indeed, in general, NP-hard.

\section{ACKNOWLEDGMENTS}

This work was supported in part by NSF grant HRD0734825 and by Grant 1 T36 GM078000-01 from the National Institutes of Health.

The author is thankful to the anonymous referees for valuable suggestions.

\section{REFERENCES}

[1] S. Ferson, "Using approximate deconvolution to estimate cleanup targets in probabilistic risk analyses", In: P. T. Kostecki, E. J. Calabrese, and M. Bonazountas (eds.), Hydrocarbon Contaminated Soils, Amherst Scientific Publishers, Amherst, Massachusetts, 1995, pp. 245-254.

[2] S. Ferson. RAMAS Risk Calc 4.0. CRC Press, Boca Raton, Florida, 2002.

[3] S. Ferson, V. Kreinovich, and W. T. Tucker, "Untangling equations involving uncertainty: deconvolutions, updates, and backcalculations", Proceedings of the NSF Workshop on Reliable Engineering Computing, Savannah, Georgia, September 15-17, 2004.

[4] S. Ferson and T. F. Long, "Deconvolution can reduce uncertainty in risk analyses", In: M. Newman and C. Strojan (eds.), Risk Assessment: Measurement and Logic, Ann Arbor Press, Ann Arebor, Michigan, 1997.

[5] G. Klir and B. Yuan, Fuzzy sets and fuzzy logic: theory and applications. Prentice Hall, Upper Saddle River, New Jersey, 1995.

[6] H. T. Nguyen and E. A. Walker, A first course in fuzzy logic, CRC Press, Boca Raton, Florida, 2005.

[7] C. H. Papadimitriou, Computational Complexity, Addison Wesley, 1993.

[8] W. T. Tucker and S. Ferson, "Setting cleanup targets in a probabilistic assessment", In: S. Mishra (ed.), Groundwater Quality Modeling and Management under Uncertainty, American Society of Civil Engineers, Reston, Virginia, 2003.

[9] R. C. Williamson and T. Downs, "Probabilistic arithmetic I: Numerical methods for calculating convolutions and dependency bounds", International Journal of Approximate Reasoning, 1990, Vol. 4, pp. 89-158. 